\title{
Glycemic impact of intensified self monitoring of blood glucose in insulin treated subjects with type 2 diabetes mellitus
}

\author{
Ejiofor T. Ugwu, Ibrahim D. Gezawa ${ }^{1}$, Olufunmilayo O. Adeleye ${ }^{2}$ \\ Department of Internal Medicine, Enugu State University of Technology, Enugu, ${ }^{1}$ Bayero University, Kano, \\ ${ }^{2}$ Lagos State University, Lagos, Nigeria
}

\begin{abstract}
Objective: The aim of this study was to evaluate if intensified self-monitoring of blood glucose (SMBG) improves glycemic control in insulin-treated subjects with type 2 diabetes mellitus (T2DM).

Methods: Ninety-six adults with stable but poorly controlled [glycated hemoglobin (HbA1c) level > 7.5\%] T2DM receiving twice-daily biphasic insulin were randomly assigned to either of the two groups: intensive monitors (IM) that performed SMBG at least twice daily or conventional monitors (CM) that performed SMBG at their discretionary frequency for 12 weeks. Demographic, clinical, and glycemic indices, including fasting blood glucose (FBG), postprandial glucose (PPG), and $\mathrm{HbA1c}$, were compared at baseline and week 12 .

Results: A total of 71 subjects ( $25 \mathrm{IM}$ and $46 \mathrm{CM}$ ) completed the study. Both groups had similar HbA1c at baseline. From baseline to week 12 , the IM group had higher mean daily test strip usage $(P<0.001)$, engaged in more frequent insulin dose adjustments $(P<0.001)$, and attained greater daily insulin dosage $(P=0.002)$. All glycemic indices including FBG, PPG, and HbA1c improved significantly from baseline to week 12 in the IM but not in the CM group. HbA1c level declined by $-1.2 \pm 0.4 \%$ in the IM group $(P=0.002)$. There was no difference in the frequency of hypoglycemia in both groups. The monthly cost of intensified SMBG was nearly four times that of conventional monitoring $(P<0.001)$.

Conclusion: Both short- and long-term glycemic control significantly improved by intensified SMBG in stable but poorly controlled insulin-treated adults with T2DM. Intensified SMBG enabled better self-titration of insulin and probably other self-care practices. This benefit, however, occurred at the expense of costs that may be difficult to sustain in resource-poor countries.
\end{abstract}

Keywords: Blood glucose, insulin, Nigeria, self-monitoring, type 2 diabetes mellitus

Address for correspondence: Dr. Ejiofor Theophilus Ugwu, Department of Internal Medicine, Enugu State University of Technology, Enugu, Nigeria.

E-mail: ofornet@yahoo.com

Received: 5.2.2017, Accepted: 29.10.2017

\section{INTRODUCTION}

Self-monitoring of blood glucose (SMBG) is now generally accepted as an integral aspect of modern diabetes care. It refers to the personalized periodic measurement of blood

\begin{tabular}{|l|l|}
\hline \multicolumn{2}{|c|}{ Access this article online } \\
\hline Quick Response Code: & Website: \\
\cline { 1 - 2 } & www.joshd.net \\
\cline { 2 - 3 } & DOI: \\
& 10.4103/joshd.J_Soc_Health_ \\
& Diabetes_7_17 \\
\hline
\end{tabular}

glucose by the individuals with diabetes, and this is most often carried out by testing the blood sample obtained by finger prick, using a point-of-care blood glucose meter. The goal of SMBG is to provide periodic information

This is an open access journal, and articles are distributed under the terms of the Creative Commons Attribution-NonCommercial-ShareAlike 4.0 License, which allows others to remix, tweak, and build upon the work non-commercially, as long as appropriate credit is given and the new creations are licensed under the identical terms.

For reprints contact: reprints@medknow.com

How to cite this article: Ugwu ET, Gezawa ID, Adeleye OO. Glycemic impact of intensified self monitoring of blood glucose in insulin treated subjects with type 2 diabetes mellitus. J Soc Health Diabetes 2018;6:27-33. 
about glycemic excursions and guide both patients and their health-care providers in determining the efficacy of diabetes care, assessing the need for treatment modification, and recognizing asymptomatic hypo- or hyperglycemia, and to empower patients in making appropriate day-to-day decisions regarding their diet, exercise, or insulin dose adjustment. ${ }^{[1,2]}$

One of the ultimate goals of effective diabetes care is to achieve sustained glycemic control, which has been demonstrated to significantly reduce the risk of development and progression of complications in both type 1 diabetes mellitus (T1DM) and type 2 diabetes mellitus (T2DM). ${ }^{[3,4]}$ Besides lifestyle modification and pharmacotherapy, the role of SMBG in achieving good glycemia in subjects with T1DM has been conclusively demonstrated and is therefore considered an integral part of intensive treatment strategy. ${ }^{[3,5]}$ Most studies evaluating the glycemic benefit of SMBG in subjects with T2DM have focused on non-insulin-treated subjects and have yielded inconsistent results, thereby making the subject an ongoing debate..$^{[6-9]}$

Despite the paucity of evidence of the utility of SMBG in improving glycemia in insulin-treated subjects with T2DM, and despite the heterogeneous nature of this group of patients, most professional guidelines endorse regular SMBG for all insulin-treated subjects with T2DM..$^{[1,2,10]}$ Consensus on the optimum testing frequency and timing is, however, lacking. Although the American Diabetes Association recommends daily testing frequency of 6-10 times in patients being treated with multiple daily insulin regimens and 1-2 times in those receiving less-intense insulin regimens such as basal insulin, it emphasizes that testing frequency should be generally based on individual needs. ${ }^{[2]}$ The Diabetes Association of Nigeria recommends SMBG at a frequency of 1-3 times daily in insulin-treated T2DM subjects. ${ }^{[10]}$ However, to the best of our knowledge, there are no published data evaluating the glycemic impact of SMBG in insulin-treated subjects with T2DM in Nigeria till date. Furthermore, data on this subject globally are grossly inadequate. This study therefore aimed at evaluating the short- and long-term glycemic impact of intensified SMBG in insulin-treated patients with T2DM.

\section{SUBJECTS AND METHODS}

\section{Study design and subjects' recruitment}

This study was conducted at the diabetes clinic of Enugu State University Teaching Hospital (ESUTH), Enugu, Nigeria. The study population consists of adults between 18 and 65 years of age with stable T2DM diagnosed according to the World Health Organization criteria. ${ }^{[1]}$ To ensure that subjects with T1DM were not recruited, participants must have a history of prior control on oral antidiabetic agents only to qualify for the study. Stability was defined as the absence of acute diabetic complications or severe chronic complications requiring hospitalization such as advanced diabetic foot ulcer, renal, or cardiac disease. Inclusion criteria were treatment with biphasic human or analog insulin with or without metformin, poor glycemic control [glycated hemoglobin (HbA1c) level >7.5\%], ownership of a functional blood glucose meter, and practicing SMBG at baseline. Pregnant women and subjects with significant visual or memory deficits were excluded.

Participants were consecutively assessed for eligibility as they presented to the diabetes clinics between November 2015 and February 2016. Potentially eligible subjects, who consented to participate in the study, were required to pay a pre-study clinic visit during which their blood glucose meters were checked for functionality and their meters were recalibrated using control solution provided by the manufacturer. Venous blood samples were collected for baseline HbA1c measurements, which were performed with i-CHROMA ${ }^{\mathrm{TM}} \mathrm{HbA1c}$ analyzer (Boditech Med, Korea). Subjects who had HbA1c level above $7.5 \%$ were enrolled in the study in a subsequent follow-up clinic visit 2 weeks later.

After documenting their sociodemographic and relevant clinical data including body mass index (BMI), subjects were interviewed on their current SMBG frequency, knowledge about self-titration of insulin, and current daily insulin dosage. All subjects were educated on the proper use of glucose meters including proper testing procedure, meter calibration, and care. They equally received education on diet and exercise, proper insulin injection techniques, selfadjustment of insulin dosages using a simple algorithm, as well as detection and management of hypoglycemia. Their baseline fasting blood glucose (FBG) and 2-h postprandial (breakfast) glucose (PPG) levels were measured with AccuChek Active glucose meter (Roche Diagnostics, Germany) and documented.

By simple random methods, participants were assigned either of the two SMBG frequency groups: intensive monitors (IM) and conventional monitors (CM). Subjects in the IM group were instructed to perform SMBG at least twice daily, including daily FBG and at least one PPG, whereas the frequency of SMBG in the CM group was left at their discretion. The research and ethics committee of ESUTH approved the protocol. 
Study end points and statistical analysis

Participants were instructed to keep record of the number of tests they performed each day, number of times they adjusted their insulin dose, and episodes of hypoglycemia, defined as either symptoms suggestive of hypoglycemia that resolved after ingestion of carbohydrate and/or documented blood glucose level less than $70 \mathrm{mg} / \mathrm{dL}$. Follow-up clinic visits were scheduled at $2,4,8$, and 12 weeks after recruitment to assess compliance to the protocol and reinforce educational strategies. At the last visit, the total daily insulin doses for each subject, and mean FBG and PPG in the past 7 days were recorded while venous blood sample was collected for repeat $\mathrm{HbA} 1 \mathrm{c}$ determination. The monthly cost of SMBG was computed by multiplying the number of tests per month by the unit cost of strips (about $\$ 0.2$ ) and taking the mean of the 3 months.

Data were analyzed with the software Statistical Package for Social Sciences (SPSS), version 20 (SPSS, Chicago, Illinois). Numbers and percentages or mean and standard deviations were computed for categorical and continuous variables, respectively. Comparison between intensive and conventional SMBG groups was made by $\chi^{2}$-test for categorical variables and by unpaired Student's $t$-test for continuous variables as appropriate. Statistical significance was established at a $P$ value of $<0.05$.

\section{RESULTS}

Baseline characteristics of the participants

A total of 96 subjects, 45 in the IM and 51 in the $\mathrm{CM}$ arm, were enrolled into the study. However, data for 71 subjects $(74.0 \%)$ were available at study end and considered suitable for analysis. Of the 45 subjects in the IM group, 25 (55.6\%) completed the study, 13 subjects were withdrawn for substantial noncompliance to the study protocol, whereas 7 subjects did not honor the scheduled clinic visits. Forty-six of the 51 subjects in the CM group $(90.2 \%)$ completed the study, one participant had cerebrovascular disease in the third week and was excluded, whereas four subjects were lost to follow-up at different stages of the study. There were no significant baseline differences in sociodemographic and clinical characteristics of the participants in the two groups [Table 1]. Baseline HbA1c values were comparable between the subjects in the IM and CM groups $(8.2 \pm 1.7 \%$ vs. $8.4 \pm 1.8 \%$, respectively; $P=0.679$ ).

Table 1: Subjects' characteristics at baseline

\begin{tabular}{|c|c|c|c|c|}
\hline Variable & Intensive $(n=25)$ & Conventional $(n=46)$ & $\chi^{2} / t$ & $P$ value \\
\hline Age (years) & $49.4 \pm 8.3$ & $48.8 \pm 9.5$ & 0.265 & 0.792 \\
\hline Gender (male) & $11(45.8)$ & $13(54.2)$ & 1.793 & 0.181 \\
\hline Level of education (post primary) & $20(40.8)$ & $29(59.2)$ & 2.178 & 0.140 \\
\hline $\mathrm{BMI}\left(\mathrm{kg} / \mathrm{m}^{2}\right)$ & $25.9 \pm 4.1$ & $27.1 \pm 5.5$ & 0.955 & 0.343 \\
\hline DM duration (years) & $5.8 \pm 2.7$ & $5.7 \pm 3.5$ & 0.124 & 0.902 \\
\hline Daily SMBG frequency & $1.0 \pm 0.3$ & $1.1 \pm 0.6$ & 0.780 & 0.438 \\
\hline Baseline daily insulin dose (IU) & $22.4 \pm 10.0$ & $24.7 \pm 9.3$ & 0.969 & 0.336 \\
\hline Baseline FBG (mg/dL) & $166 \pm 24.4$ & $159.5 \pm 33.1$ & 0.862 & 0.392 \\
\hline Baseline PPG (mg/dL) & $194 \pm 49.8$ & $201 \pm 28.0$ & 0.760 & 0.450 \\
\hline Baseline $\mathrm{HbA} 1 \mathrm{c}(\%)$ & $8.2 \pm 1.7$ & $8.4 \pm 1.8$ & 0.419 & 0.679 \\
\hline
\end{tabular}

BMI, body mass index; DM, diabetes mellitus; SMBG, self-monitoring of blood glucose; FBG, fasting blood glucose; PPG, postprandial glucose. Data are in mean \pm SD or numbers and percentages.

Table 2: Comparison between subjects in the intensive and conventional SMBG groups at study end

\begin{tabular}{lcrr}
\hline Variable & Intensive $(n=25)$ & Conventional $(n=46)$ & $\chi^{2} / t$ \\
\hline BMI $\left(\mathrm{kg} / \mathrm{m}^{2}\right)$ & $26.3 \pm 5.0$ & $27.8 \pm 4.2$ & 1.343 \\
Daily SMBG frequency & $2.9 \pm 0.7$ & $1.6 \pm 0.9$ & 0.259 \\
Number of insulin adjustments & $17.4 \pm 8.8$ & $9.5 \pm 6.3$ & $<0.001$ \\
Daily insulin dose at week 12 (IU) & $40.8 \pm 12.3$ & $32.2 \pm 9.6$ & $<0.001$ \\
Frequency of hypoglycemia & $11 \pm 6.0$ & $15 \pm 10.0$ & 0.002 \\
Mean FBG at week 12 (mg/dL) & $143 \pm 41.2$ & $152 \pm 55.6$ & 3.260 \\
Mean PPG at week 12 (mg/dL) & $163 \pm 20.5$ & $190 \pm 33.6$ & 2.339 \\
HbA1c at week 12 (\%) & $7.0 \pm 0.7$ & $8.1 \pm 1.5$ & 0.709 \\
Change in HbA1c (\%) & $20.9 \pm 13.3$ & $6.6 \pm 6.0$ & 3.658 \\
Reached glycemic goal (HbA1c $<7 \%)$ & $14(56.0)$ & $13(28.2)$ & 3.699 \\
Monthly cost of SMBG (\$) & $22.6 \pm 3.4$ & $6.7 \pm 4.9$ & 6.276 \\
\hline
\end{tabular}

SMBG, self-monitoring of blood glucose; BMI, body mass index; FBG, fasting blood glucose; PPG, postprandial glucose; HbAlc, glycated hemoglobin; $S D$, standard deviation.

Data are in mean \pm SD or numbers and percentages. 
Comparison between the intensive and the conventional SMBG monitors at study end

Table 2 shows the clinical and biochemical parameters of both the study groups at the end of follow-up. Subjects in the IM group performed more frequent SMBG, using an average of $2.9 \pm 0.7$ test strips per day compared to $1.6 \pm 0.9$ test strips in the CM group $(P<0.001)$. The IM group also engaged in more frequent insulin adjustments than the subjects in the CM group $(P<0.001)$. This resulted in a greater increment in the daily insulin dosage in the IM than the CM group from baseline. Consequently, the week 12 daily insulin dose was significantly higher in the IM group (40.8 \pm 12.3 vs. $32.2 \pm 9.6$ units; $P=0.002$ ). Although subjects in the CM group had more episodes of hypoglycemia compared to the IM group, this difference did not reach statistical significance $(P=0.072)$. Mean FBG did not differ significantly between the two groups at study end. In contrast, week 12 mean PPG was significantly lower in the IM than in the CM group (IM, $163.0 \pm 20.5 \mathrm{mg} / \mathrm{dL}$; CM, $190.0 \pm 33.6 \mathrm{mg} / \mathrm{dL} ; P=0.001)$.

Long-term glycemic control was significantly better in the IM group. In this group, $56.0 \%$ of the subjects achieved

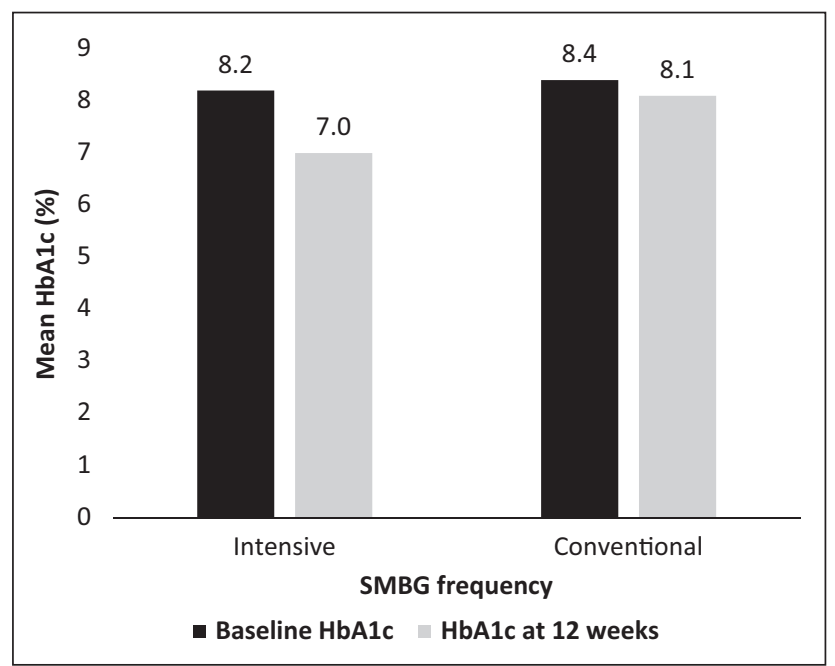

Figure 1: Changes in $\mathrm{HbA1c}$ from baseline to week 12 in the intensive and conventional groups target $\mathrm{HbA} 1 \mathrm{c}$ value of $<7 \%$ compared to $28.2 \%$ of the subjects in the CM group who achieved a similar outcome $(P=0.002)$. The week 12 mean HbA1c level in the IM group was significantly lower compared to the $\mathrm{CM}$ group $(7.0 \pm 0.7 \%$ vs. $8.1 \pm 1.5 \% ; P=0.001)$. Figure 1 shows the changes in mean $\mathrm{HbA} 1 \mathrm{c}$ level from baseline to week 12 in both the study groups.

\section{Within group changes from baseline to week 12}

Within-group changes in body proportions, SMBG frequencies, insulin dosages, and glycemic indices from baseline to week 12 in both the study arms are shown in Table 3. BMI did not change significantly in both the groups. Daily insulin dosage increased significantly in both the groups from baseline to week 12 . However, the increment was significantly higher in IM than in the CM group (18.4 \pm 3.2 vs. $7.5 \pm 2.0$ units; $P<0.001$ ). Significant decline in all the indices of glycemic control from baseline to week 12 occurred in the IM but not in the CM group. In the former, FBG declined by $23.0 \pm 9.6 \mathrm{mg} / \mathrm{dL}$ and PPG by $31.0 \pm 10.8 \mathrm{mg} / \mathrm{dL}$. The IM group achieved a significant reduction in $\mathrm{HbA} 1 \mathrm{c}$ level by $-1.2 \pm 0.4 \%$ representing nearly $21 \%$ decline from the baseline in contrast to the nonsignificant decline of $0.3 \pm 0.4 \%$ in the conventional group.

\section{Costs of SMBG in each group}

The IM group spent nearly four times as much as the CM group per month on test strip usage $(P<0.001)$. Excluding the cost of blood glucose meter, each subject in the intensive group spent an average of $\$ 22.6 \pm 3.4$ per month.

\section{DISCUSSION}

When the American Diabetes Association released its first recommendation in 1982 regarding SMBG in T2DM, little did it know that it was going to generate much controversy. Unlike T1DM, in which the benefit of intensified SMBG is unequivocal, ${ }^{[3,5]}$ evidences are insufficient to tilt the argument completely in favor of frequent SMBG in T2DM, and this consequently stimulated research interest

Table 3: Within-group differences from baseline to week 12 in the intensive and conventional SMBG groups

\begin{tabular}{|c|c|c|c|c|c|c|c|c|}
\hline \multirow[t]{2}{*}{ Variable } & \multicolumn{4}{|c|}{ Intensive $(n=25)$} & \multicolumn{4}{|c|}{ Conventional $(n=46)$} \\
\hline & Baseline & Week 12 & Mean difference ${ }^{*}$ & $P$ value & Baseline & Week 12 & Mean difference* & $P$ value \\
\hline BMI $\left(\mathrm{kg} / \mathrm{m}^{2}\right)$ & $25.9 \pm 4.1$ & $26.3 \pm 5.0$ & $0.4 \pm 1.3$ & NS & $27.1 \pm 5.5$ & $27.8 \pm 4.2$ & $0.7 \pm 1.0$ & NS \\
\hline Daily testing frequency & $1.0 \pm 0.3$ & $2.9 \pm 0.7$ & $1.9 \pm 0.2$ & $<0.001$ & $1.1 \pm 0.6$ & $1.6 \pm 0.9$ & $0.5 \pm 0.2$ & 0.002 \\
\hline Daily insulin dose (IU) & $22.4 \pm 10.0$ & $40.8 \pm 12.3$ & $18.4 \pm 3.2$ & $<0.001$ & $24.7 \pm 9.3$ & $32.2 \pm 9.6$ & $7.5 \pm 2.0$ & $<0.001$ \\
\hline Mean FBG (mg/dL) & $166 \pm 24.4$ & $143 \pm 41.2$ & $-23.0 \pm 9.6$ & 0.020 & $159.5 \pm 33.1$ & $152 \pm 55.6$ & $-7.5 \pm 9.5$ & NS \\
\hline Mean PPG (mg/dL) & $194 \pm 49.8$ & $163 \pm 20.5$ & $-31.0 \pm 10.8$ & 0.006 & $201 \pm 28.0$ & $190 \pm 33.6$ & $-11.0 \pm 6.5$ & NS \\
\hline HbA1c (\%) & $8.2 \pm 1.7$ & $7.0 \pm 0.7$ & $-1.2 \pm 0.4$ & 0.002 & $8.4 \pm 1.8$ & $8.1 \pm 1.5$ & $-0.3 \pm 0.3$ & NS \\
\hline
\end{tabular}

SMBG, self-monitoring of blood glucose; BMI, body mass index; FBG, fasting blood glucose; PPG, postprandial glucose; HbAlc, glycated hemoglobin; *, week 12 minus baseline; NS, not significant; SD, standard deviation.

Data are in mean \pm SD or numbers and percentages. 
Ugwu, et al.: Intensive glucose monitoring and glycemic outcome in insulin treated type 2 diabetes

in this direction. Unfortunately, most of these researches have focused on subjects on oral agents. Although widely recommended by nearly all professional groups, ${ }^{[1,2,10]}$ the value of intensified SMBG in insulin-treated subjects with T2DM remains mainly speculative and largely extrapolated from its proven benefit in T1DM rather than from robust evidence. The cost of intensified SMBG is prohibitive. ${ }^{[12,13]}$ Therefore, the quest to justify its use in stable insulin-treated subjects with T2DM, who are not on multidose insulin regimen, is justifiable.

Stimulated by the nonexistence of local data and paucity of global data on this subject, we conducted this longitudinal study. We prospectively followed up 71 poorly controlled subjects who were being treated with twice-daily biphasic insulin-made up of 25 subjects who monitored their blood glucose at least twice daily including a daily fasting glucose and at least one daily PPG and another 46 appropriately matched cohort who monitored their blood glucose at their own discretion. To minimize confounders, we educated both the groups on proper SMBG technique, insulin dosage adjustment using simple algorithm, and the need to adhere to dietary and exercise regimens.

The findings from this study strongly suggest that intensive SMBG impacts positively on both short- and long-term glycemic control in stable insulin-treated subjects with T2DM. We observed that SMBG at a frequency of at least twice daily resulted in reaching glycemic target in $56 \%$ of the subjects and led to a significant reduction in $\mathrm{HbA} 1 \mathrm{c}$ levels by $-1.2 \%$. These findings are supported by previous observational and randomized controlled trials. ${ }^{[14-16]}$ In the Diabetes Outcomes in Veterans Study (DOVES), one of the few prospective studies that have evaluated the benefit of intensified SMBG on glycemic control in T2DM subjects on insulin treatment, 201 stable T2DM participants performed SMBG at least four times daily including before every meal and at bedtime for 8 weeks and subsequently followed up for 52 weeks on usual care. ${ }^{[14]}$ During the intensive monitoring period, there was a significant reduction in $\mathrm{HbA} 1 \mathrm{c}$ level from baseline by $0.30 \pm 0.68 \%$ at 4 weeks to $0.36 \pm 0.88 \%$ at 8 weeks $(P=0.001$, respectively). Regression analysis showed that compliance with the SMBG protocol influenced the week $8 \mathrm{HbA1c}$ level more than age, sex, BMI, exercise level, carbohydrate consumption, or baseline $\mathrm{HbA} 1 \mathrm{c}$ level. Interestingly, significant decline in HbA1c level $(0.31 \pm 1.17 \%, P=0.001)$ persisted in the 159 subjects followed up for 52 weeks on usual care. ${ }^{[14]}$ Although findings from the DOVES clearly suggest glycemic benefit of intensified SMBG in insulin-treated T2DM, it is noteworthy that subjects in the intervention group performed SMBG four times daily. This frequency may be difficult to adapt in real life, especially in resource-poor countries such as sub-Saharan Africa. Ugwu et al. ${ }^{[13]}$ recently reported that only $7.9 \%$ of subjects with T2DM in Eastern Nigeria performed SMBG at least once daily. Even in developed countries with higher income, it has been shown that the real-life mean blood glucose testing frequency in insulin-treated T2DM patients ranges from 1.7 tests/day in Spain to 3 tests/day in Canada. ${ }^{[17,18]}$ Considering that the subjects in the DOVES were on basal insulin, four times daily SMBG frequency appears to be an "overprescription" and in conflict with most guidelines. Our study is therefore more applicable to clinical care.

Some studies have, on the other hand, contradicted our findings. ${ }^{[7,19]}$ In the Fremantle Diabetes Study, Davis et al. ${ }^{[7]}$ observed no cross-sectional or longitudinal association between SMBG frequency and HbA1c level in both insulin-treated and non-insulin-treated subjects with T2DM. It is noteworthy, however, that this quoted study was not properly designed to elicit any glycemic benefit of SMBG. First, the insulin-treated subjects were highly heterogeneous, as they were being treated with varied insulin types and injection frequencies. Second, there was neither a standard SMBG protocol nor a comparator group against which the intervention was tested. Third, there was no plan to incorporate SMBG results into diabetes self-care such as intensification of lifestyle or insulin dose adjustment. It had been demonstrated that SMBG is more or less an exercise in futility if results obtained are not integrated into clinical care. ${ }^{[19]}$ In another population-based study, in which $39 \%$ of insulin-treated subjects performed SMBG at least once daily and others less frequently, no significant relationship was observed between testing frequency and HbA1c level. ${ }^{[20]}$ This quoted study is also limited by its cross-sectional design.

It appears that the long-term glycemic benefit of intensified SMBG is indirect, being predominantly mediated through behavioral changes that improve diabetes self-management such as better adherence to diet and exercise regimen, improved drug compliance, medication adjustment, and more frequent contact with diabetes health-care providers. An aspect of this influence of SMBG on diabetes self-care behavior was demonstrated in this study. We observed that subjects in the intensive SMBG group made more frequent insulin dosage adjustments than the conventional group $(P<0.001)$. Consequently, week 12 insulin dosage was significantly higher in the intensive than the conventional group. Although we do not have information about the dietary and exercise behavior of our study participants, it is unlikely that these lifestyle factors were different between the two study groups as both received equal counseling at 
baseline on these lifestyle issues. Furthermore, BMI was not significantly different between the two groups at the end of the study nor changed significantly within either group from the baseline. One cannot, however, completely rule out the possibility that intensive monitoring might have stimulated greater adherence to lifestyle regimen as have been demonstrated in the past. ${ }^{[1]}$

The impact of PPG on HbA1c level was also highlighted in this study. We observed that although the intensive group had a significantly lower $\mathrm{HbA1}$ c level from baseline to week 12 than the conventional group, there was no significant difference in week 12 mean FBG in both the groups. However, the intensive SMBG group achieved much more decline in PPG from baseline $(P=0.006)$ compared to the nonsignificant decline in PPG in the conventional group. It therefore appears that the significant difference in week 12 $\mathrm{HbA1c}$ in both groups was accounted for, predominantly, by the differences in PPG control. Our study thus supports previous assertions that PPG is a greater contributor to $\mathrm{HbA1c}$ level than fasting glucose level. ${ }^{[22,23]}$

Besides its impact on long-term glycemic control, frequent SMBG is also widely advocated as a significant player in the prevention and early detection of hypoglycemia, especially asymptomatic events. ${ }^{[1,2,24]}$ In contrast, our study did not show any benefit of intensified SMBG in reducing the frequency of hypoglycemia, and this is supported by a recent randomized controlled trial. ${ }^{[25]}$ However, although not statistically significant, we observed a trend toward lower frequency of hypoglycemia in the intensive monitoring group ( $P=0.072$ ), despite this group attaining a higher daily insulin dosage from baseline to week 12. Like the present work, most studies reporting on the relationship between SMBG and frequency of hypoglycemia have relied on the subjects' self-reported events and are therefore bound to generate conflicting results because of the errors inherent in such method. On the one hand, frequent SMBG is expected to engender more appropriate and timely adjustments in lifestyle and medications leading to lower frequency of hypoglycemia. On the other hand, it may also be associated with higher frequency of hypoglycemia owing to increased detection of asymptomatic events rather than biochemical difference between the intervention group and comparators as had been observed in one study. ${ }^{[8]}$

Improvement in glycemic control in the intensive SMBG group, which was observed in this study, was not without economic consequences. The monthly cost of SMBG in the intensive group was much higher, averaging nearly $\$ 23$ per month. This cost may be unsustainable in low- and middle-income countries with lean resources. In Nigeria, for instance, an average person lives below the poverty line of $<\$ 60$ per month. ${ }^{[26]}$ Studies have consistently highlighted the huge costs associated with SMBG. ${ }^{[12,13,17,18]}$ In 2011, the National Health Service in the United Kingdom reportedly spent $£ 158$ million on SMBG, representing about $21 \%$ of the diabetes treatment costs. ${ }^{[12]}$ It is estimated that an equivalent of about $\$ 126$ million is spent on SMBG annually in Nigeria. ${ }^{[13]}$ Nevertheless, as SMBG-mediated intensification of treatment in T2DM results in good glycemic control and consequent reduction in microvascular complications, SMBG is said to be cost-effective. ${ }^{[4,16]}$

\section{CONCLUSION}

In conclusion, our study clearly demonstrated that intensified SMBG in stable insulin-treated subjects with T2DM benefits both short- and long-term glycemic control, although at the expense of increased health-care costs that are capable of inhibiting its applicability in real life, particularly, in resource-poor countries.

Our study has some limitations. Our sample size is too small to allow for extrapolation of these findings to the general population. Second, all our subjects were on twice-daily premix insulin. Our data cannot therefore be applied to T2DM subjects on other insulin regimen. Moreover, data for nearly half of the subjects assigned to the intensive group could not be analyzed because of substantial noncompliance with the study protocol and attrition. The extent to which this might have affected our findings is unknown. We recommend a larger, properly designed randomized controlled trial that will provide better clarification on this important subject.

Financial support and sponsorship

Nil.

\section{Conflicts of interest}

There are no conflicts of interest.

\section{REFERENCES}

1. IDF Clinical Guidelines Task Force: Global Guideline for Type 2 Diabetes. 2012. Available from: www.idf.org/sites/default/files/ IDF\%20T2DM\%20Guideline.pdf. [Last accessed on 2016 Oct 15].

2. American Diabetes Association. Glycaemic targets. Sec. 5. In: Standards of Medical Care in Diabetes 2016. Diabetes Care 2016;9(Suppl 1): S39-46.

3. Diabetes Control and Complications Research Group. The effect of intensive treatment of diabetes on the development and progression of long-term complications in insulin-dependent diabetes mellitus. N Engl J Med 1993;329:977-86.

4. UK Prospective Diabetes Study (UKPDS) Group. Intensive blood-glucose control with sulphonylureas or insulin compared with 
conventional treatment and risk of complications in patients with type 2 diabetes (UKPDS 33). Lancet 1998;352:837-53.

5. Miller KM, Beck RW, Bergenstal RM, Goland RS, Haller MJ, McGill $\mathrm{JB}$, et al. Evidence of a strong association between frequency of self monitoring of blood glucose and hemoglobin A1c levels in T1D exchange clinic registry participants. Diabetes Care 2013;36:2009-14.

6. Clar C, Barnard K, Cummins E, Royle P, Waugh N. Aberdeen Health Technology Assessment Group. Self-monitoring of blood glucose in type 2 diabetes: Systematic review. Health Technol Assess 2010;14: $1-140$.

7. Davis WA, Bruce DG, Davis TM. Does self-monitoring of blood glucose improve outcome in type 2 diabetes? The Fremantle Diabetes Study. Diabetologia 2007;50:510-5.

8. Guerci B, Drouin P, Grange V, Bougneres P, Fontaine P, Kerlan V, et al.; The ASIA Group. Self-monitoring of blood glucose significantly improves metabolic control in patients with type 2 diabetes mellitus: The Auto-Surveillance Intervention Active (ASIA) study. Diabetes Metab 2003;29:587-94.

9. Soumerai SB, Mah C, Zhang F, Adams A, Barton M, Fajtova V, et al. Effects of health maintenance organization coverage of self-monitoring devices on diabetes self-care and glycaemic control. Arch Intern Med 2004;164:645-52.

10. Chinenye S, Ofoegbu EN, Onyemelukwe GC, Uloko AE, Ogbera AO. In: Clinical Practice Guidelines for Diabetes Management in Nigeria. $2^{\text {nd }}$ ed. Diabetes Association of Nigeria; 2013. pp. 12-3.

11. World Health Organization. Definition, Diagnosis and Classification of Diabetes Mellitus and Its Complications. Report of WHO Consultation. WHO/NCD/NCS/99. Geneva: WHO;1999. pp. 1-58.

12. National Prescribing Centre. Data focused commentary: Type 2 diabetes: Self monitoring of blood glucose. 2012. Available from: http://www.npc.nhs.uk/therapeutics/cardio/ diabetes_2/ resources/dfc_self_monitoring_of_blood_glucose.pdf. [Last accessed on 2017 Jan 5].

13. Ugwu ET, Orjioke CJG, Young EE. Self monitoring of blood glucose among patients with type 2 diabetes mellitus in eastern Nigeria: Need for multi-strategic interventions. Curr Diabetes Rev 2016 Oct 14. DOI: 10.2174/1573399812666161014111618. [Epub ahead of print].

14. Murata C, Shah JH, Hoffman RM, Wendel CS, Adam KD, Solvas PA, et al. Intensified blood glucose monitoring improves glycaemic control in stable, insulin-treated veterans with type 2 diabetes: The Diabetes Outcomes in Veterans Study (DOVES). Diabetes Care 2003;26: 1759-63.
15. Karter AJ, Ackerson LM, Darbinian JA, D'Agostino RB Jr, Ferrara A, Liu J, et al. Self-monitoring of blood glucose levels and glycaemic control: The Northern California Kaiser Permanente Diabetes Registry. Am J Med 2001;111:1-9.

16. Ohkubo Y, Kishikawa H, Araki E, Miyata T, Isami S, Motoyoshi S, et al. Intensive insulin therapy prevents the progression of diabetic microvascular complications in Japanese patients with non-insulin-dependent diabetes mellitus: A randomized prospective 6-year study. Diabetes Res Clin Pract 1995;28:103-17.

17. Neeser K, Weber C. Cost impact of self measurement of blood glucose on complications of type 2 diabetes: The Spanish perspective. Diabetes Technol Ther 2009;11:509-16.

18. Yeaw J, Lee WC, Wolden ML, Christensen T, Groleau D. Cost of self-monitoring of blood glucose in Canada among patients on an insulin regimen for diabetes. Diabetes Ther 2012;3:7.

19. Gallichan M. Self monitoring of glucose by people with diabetes: Evidence based practice. BMJ 1997;314:964-7.

20. Harris MI. Frequency of blood glucose monitoring in relation to glycaemic control in patients with type 2 diabetes. Diabetes Care 2001;24:979-82.

21. Peel E, Parry O, Douglas M, Lawton J. Blood glucose self monitoring in non-insulin-treated type 2 diabetes: A qualitative study of patients perspectives. Br J Gen Pract 2004;54:183-8.

22. Avignon A, Radauceanu A, Monnier L. Nonfasting plasma glucose is a better marker of diabetic control than fasting plasma glucose in type 2 diabetes. Diabetes Care 1997;20:1822-6.

23. Soonthornpun S, Rattarasarn C, Leelawattana R, Setasuban W. Postprandial plasma glucose: A good index of glycaemic control in type 2 diabetic patients having near-normal fasting glucose levels. Diabetes Res Clin Pract 1999;46:23-7.

24. Farmer A, Wade A, Goyder E, Yudkin P, French D, Craven A, et al. Impact of self monitoring of blood glucose in the management of patients with non-insulin treated diabetes: Open parallel group randomised trial. BMJ 2007;335:132.

25. O'Kane MJ, Bunting B, Copeland M, Coates VE; On behalf of the ESMON study group. Efficacy of self monitoring of blood glucose in patients with newly diagnosed type 2 diabetes (ESMON study): Randomized controlled trial. BMJ 2008;336: 1174-7.

26. Central Intelligence Agency (US) report 2010. Available from: https:// www.cia.gov/library/publications/the-world-factbook/fields/2046. html. [Last accessed on 2017 Jan 8]. 